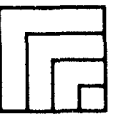

Allin

WO

3210

Association for Information and Image Management

1100 Wayne Avenue. Slitte 1100

Silver Spring Marytand 20910

$301 / 587-8202$

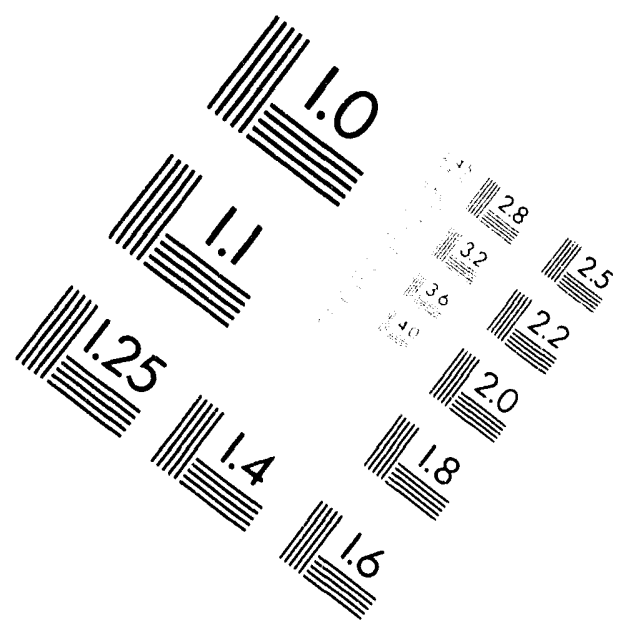

Centimeter

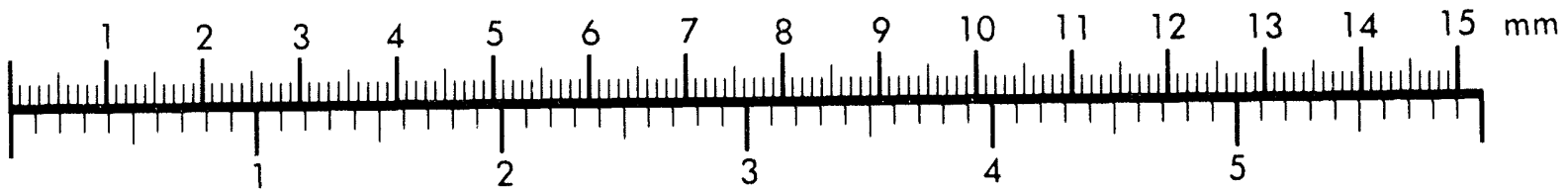

Inches
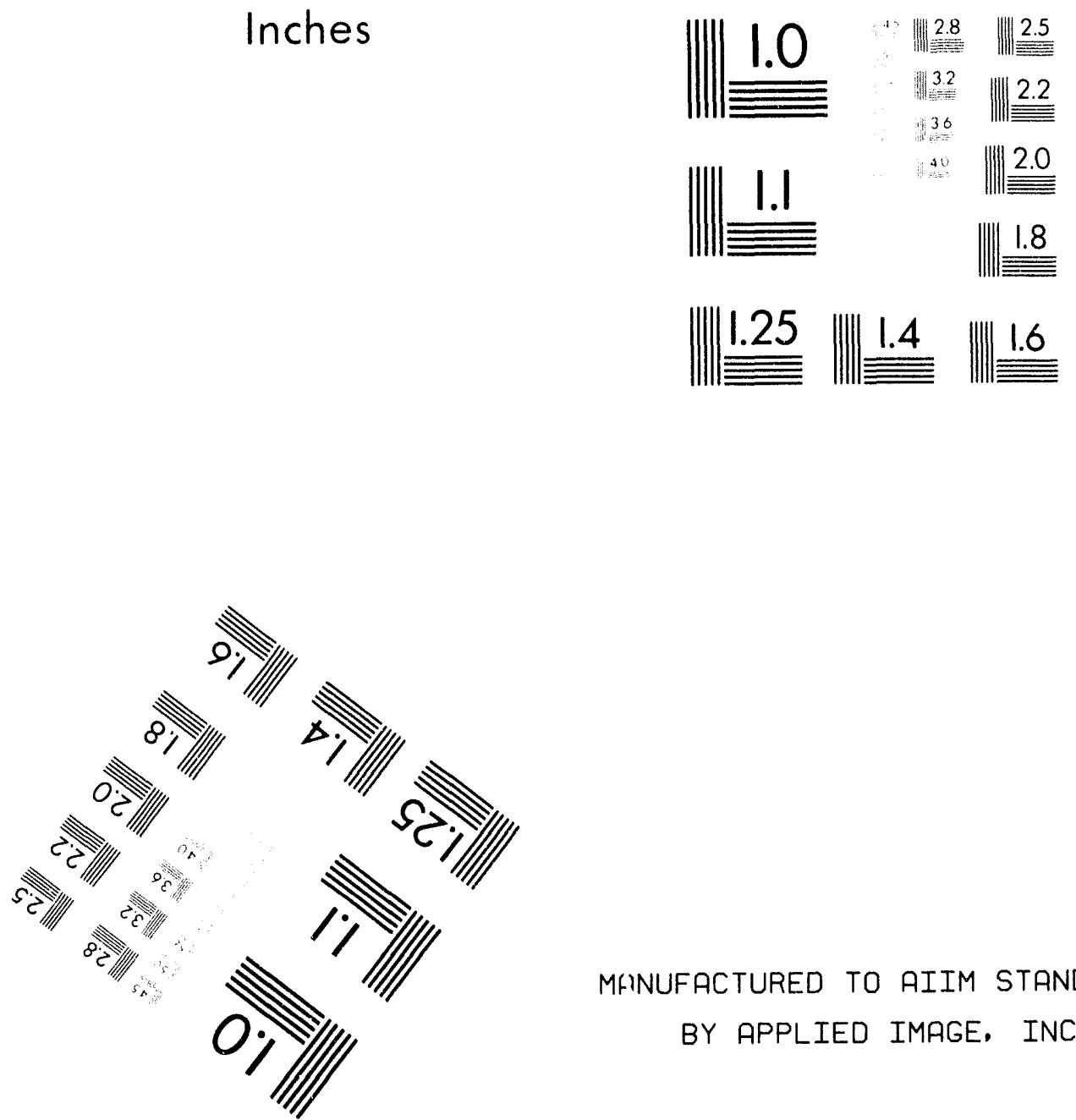

MFINUFACTURED TO AIIM STANDARDS

BY APPLIED IMAGE, INC.

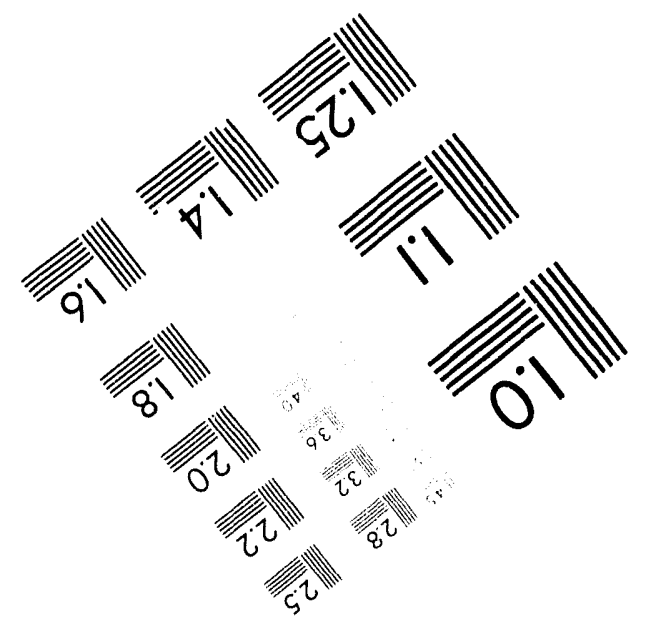



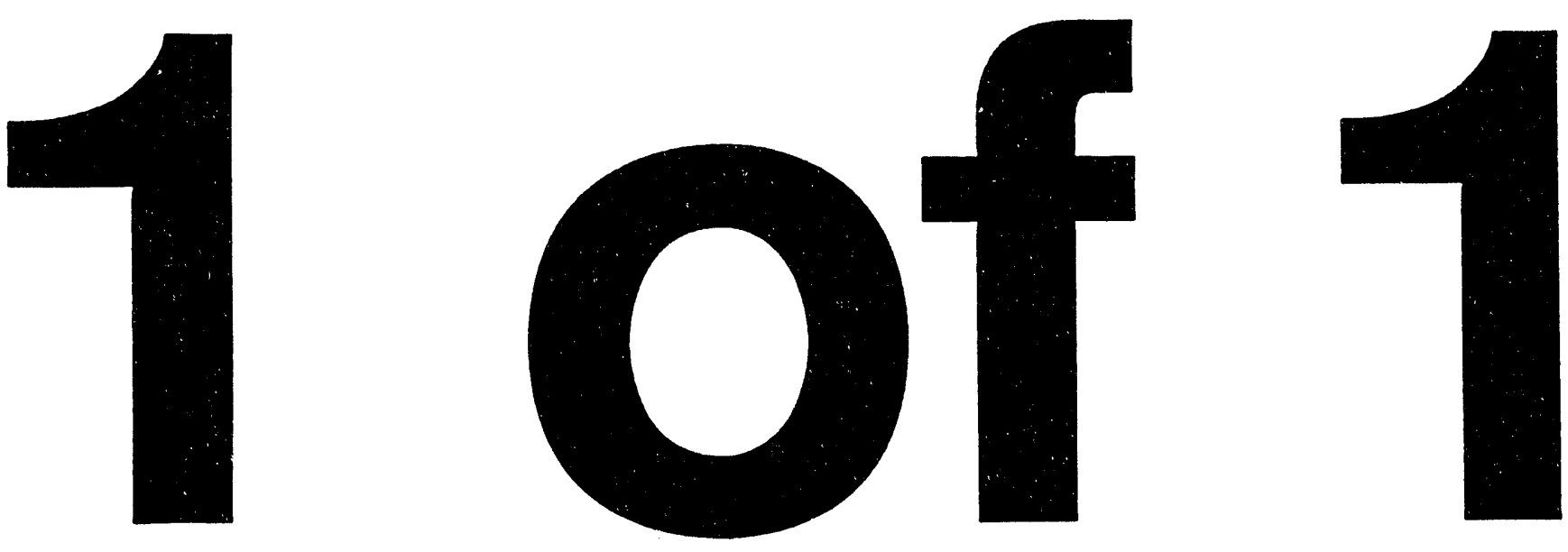


\section{Conf $940552--14$}

LA-UR- 94-1701
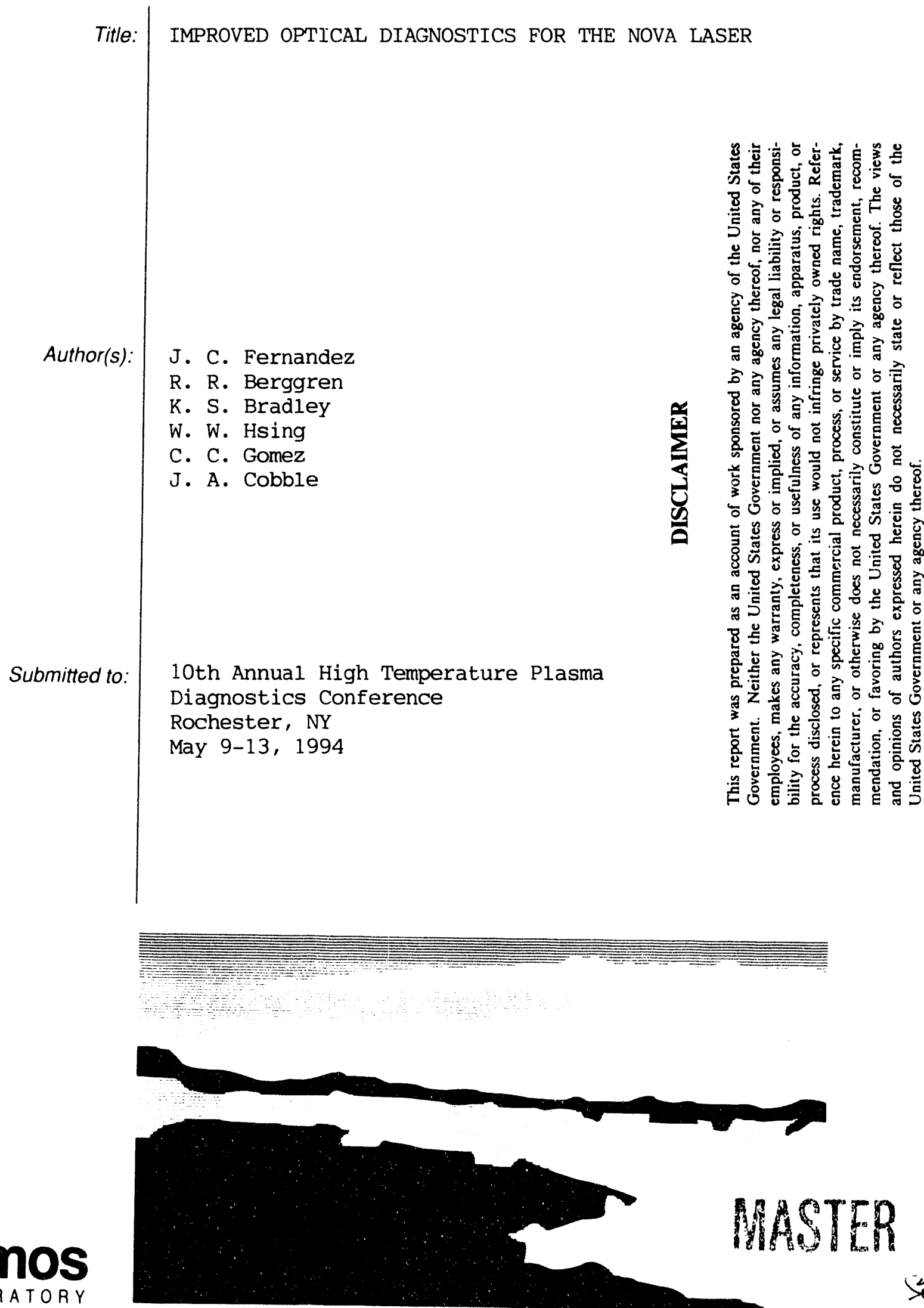


\title{
Improved Optical Diagnostics for the NOVA Laser
}

\author{
Juan C. Fernández, Ralph R. Berggren, Keith S. Bradley, \\ Warren W. Hsing, Camilo C. Gómez and James A. Cobble \\ Los Alamos National Laboratory, Los Alamos, NM 87545
}

This paper describes three diagnostics for detecting optical scatter from NOVA laser targets. Detecting such scatter can help not only our understanding of plasma instabilities in laser plasmas, but also our efforts at plasma characterization, particularly hohlraum plasmas. These diagnostics are: the Full Aperture Backscatter Station (FABS), presently being built; the Oblique Scatter Array (OSA), just starting operation; and the Axial Imager, also just starting operation. FABS will allow imaging at high resolution of Brillouin and Raman backscatter. The OSA allows a quantitative measurement of Brillouin and Raman scatter in many directions (assuming the target allows the scatter to escape). The axial imager allows high-resolution imaging of Brillouin, Two-Plasmon Decay and Raman scatter emitted towards the East direction, which is along the symmetry axis of the NOVA laser beams. 


\section{Introduction}

The airn of inertial confinement fusion (ICF) is to compress a D-T capsule and, by properly timing shocks, create a hot-spot in the center which ignites a fusion burn. In indirect-drive ICF, the capsule is driven by X-rays, made by laser beams hitting the walls of a high-Z cavity called a hohlraum.[5] The beams create a plasma blowoff which, in ignition hohlraums, could reach electron densities of $n_{c} \leq 10^{21} \mathrm{~cm}^{-3}$ and electron temperatures $T_{c} \approx 3-5 \mathrm{keV}$. The $351 \mathrm{~nm}$ laser beams drive instabilities in these plasmas, which scatter light in optical and UV wavelengths. Sufficiently high levels of scattered light could threaten the success of ignition laser systems. Gas-filled hohlraums designed by Los Alamos are simulating conditions expected in ignition hohlraums to study these instabilities. Even when the levels are not too high, scattered light can be useful for plasma characterization, which is important to benchmark hydrodynamic modeling of the target. Instabilities of interest are Stimulated Brillouin Scattering (SBS), Stimulated Raman Scattering (SRS), and two-plasmon $\left(2 \omega_{p c}\right)$ decay. These three instabilities represent, respectively, the decay of laser light into: an ion-acoustic wave and scattered light near $351 \mathrm{~nm}$; a plasma wave and 
scattered light below $702 \mathrm{~nm}$; and two plasma waves which, through other processes, create scattered light at $234 \mathrm{~nm}$.[1] SBS can occur where $n_{c} \leq 9 \times 10^{21} \mathrm{~cm}^{-3}$, SRS can occur where $n_{c} \leq 2.25 \times 10^{21} \mathrm{~cm}^{-3}$, and $2 \omega_{p c}$ can occur where $n_{c} \approx 2.25 \times 10^{21} \mathrm{~cm}^{-3}$. Figure 1 shows the illumination geometry of a typical NOVA hohlraum, along with some diagnostics used to detect the scattered light. New or improved diagnostics are highlighted. Three diagnostics are described in this paper: the Full-Aperture Backscatter Station (FABS); the Oblique-Scatter Array (OSA); and the Axial Imager.

\section{Diagnostic Description and Specifications}

FABS: This diagnostic will measure the SBS and SRS returning within the cone of the plano-convex focusing lens in beam 7. This allows the localization of the instability within the hohlraum. And by focusing on a single SRS wavelength (which fixes $n_{c}$ from which the imaged scattered light comes), the hohlraum $n_{c}$ is characterized. The design yields a spatial resolution at the target of $25 \mu \mathrm{m}$ for any chosen wavelength in the range of 349-702 $\mathrm{nm}$. The image is achromatic either over $12 \mathrm{~nm}$ around the chosen wavelength (when near $700 \mathrm{~nm}$ ) 
or over $2 \mathrm{~nm}$ (when near $351 \mathrm{~nm}$ ). The task is greatly compli cated by the presence (near the focusing lens) of a Random-Phase Plate (RPP), used to spatially smooth the beam by breaking it into small beamlets which interfere at the lens focus.[4,2] The RPP greatly distorts the backscatter. Thus, an image of the RPP must be formed, an inverse RPP placed at this image to compensate, and then the target must be imaged achromatically.

Figure 2 illustrates the main elements of the system. The last laser turning mirror in beam 7 is about $5 \%$ transmisive to the backscatter returning from the target, which is nearly collimated by the focusing lens. Primary (34" Dia.) and secondary $\left(7.5^{n}\right.$ Dia.) spherical mirrors (made out of uncoated fused silica) make an image with much chromatic aberration caused by the focusing lens. The field lens makes an achromatic image of the RPP, where the RPP compensator plate is located. All these elements are within a central obscuration which all NOVA beams have for unrelated reasons. (In the actual design, the doublet and RPP compensator are located in the horizontal plane beyond the switchout mirror.) Beyond the compensator, the combination (not shown) of a collimator lens, a second field lens and a combined 
lens/holographic optical element, creates an achromatic image of the target magnified $10 \times$. Because the pixel size of the typical gated detector ( $0.15 \mathrm{~ns}$ gate) which will be used is about $50 \mu \mathrm{m}$, the resolution will be about 5 pixels wide.

OSA: The array of 50 PIN6LC diodes measure absolute scattered energy in the $\vec{E}$ and $\vec{B}$ planes of beam 7 , often used as the probe beam in these experiments. (All NOVA beams are linearly polarized.) Figure 3 shows the diode locations on the NOVA target chamber relative to beam 7. Optical filters, chosen separately for each diode, allows discrimination between SBS and SRS, and protects the diodes from unwanted X-rays and debris from the target. In fact, multiple diodes placed at nearly the same location can provide either better statistics or complementary observations at different wavelengths. Each location has a holder capable of accommodating up to four diodes, allowing some flexibility for different experiments. Although clearly some locations are shielded from scatter from beam 7 by the hohlraum wall, other open NOVA targets allow beam-7 scatter to escape into all directions. It should be noted that while the diode spectral response around $351 \mathrm{~nm}$ is flat, it varies by about $\times 3$ in the $400-700 \mathrm{~nm}$ band. Thus they are 
not an ideal SRS diagnostic.

For two-color experiments, where plasma formation is done with $527 \mathrm{~nm}$ beams and probing is done with beam 7 at $351 \mathrm{~nm}$, interaction -beam scatter can be distinguished from heater-beam scatter simply by proper diode filtration. However, for one-color experiments, it is necessary to discriminate probe-beam scatter temporally, since the probe beam is often delayed relative to the heater beams. But these diodes hava a response time much longer than the $\approx 1 \mathrm{~ns}$ typical duration of target illumination, so they are incapable of power measurements. But ten fiber-optic light collectors (separately filtered and strategically located) feed their signal to a streak camera, which allows $\approx 0.1 \mathrm{~ns}$ time resolution.

Axial Imager: It has an $f / 8$ light-collection cone along the East direction (Fig. 1). The assembly is mounted on a cart in the recently commissioned NOVA Six Inch Manipulator (SIM) \# 7. SIM 7, mounted on the East-end of the target chamber, was designed and built by Los Alamos. Livermore did the required modifications to the space-frame and the required seismic and structural analysis. Three elements are placed in the cart: closest to the target, a fused-silica blast shield; then 
the desired filters; and finally the achromat imaging lens. These are all $3^{n}$ optics. Two achromats have been designed: one for the UV (234$450 \mathrm{~nm}$ ), and one for the visible. Presently, the UV lens is in use. Outside the SIM cart, a turning mirror directs the light towards an optical table above. There, a group of beam splitters and turning mirrors (which add about $24^{n}$ to the optical path) place four images (magnified $\approx 9 \times)$ at the four-frame gated detector. Figure 4 illustrates the setup. The optics are capable of $20-50 \mu \mathrm{m}$ resolution at the target. The lens and all the filters (except those right at the detector) are ground to a flatness of better than $\lambda / 10$ so as not to degrade the resolution.

The four-frame detector is a unit from Grant Applied Physics of Berkeley, CA (made in England), model GOU/18/4/NS. The imager is based on a microchannel plate intensified wafer tube with an S25 photocathode. The phosphor is P20. The pulser electronics allows a minimum 0.12 ns exposure in each of the four detectors, each at an arbitrarily chosen time (within a 0.05 ns jitter). Each detector has an active-area dimension of $18 \mathrm{~mm}$.[3] 


\section{Diagnostic Status and Results}

FABS: Presently, the optical designs for both NOVA focusing lenses, $f / 4$ and $f / 8$, have been completed. The tolerance analysis necessary to fabricate the lenses is near completion. The $34^{\text {" primary mirror has }}$ been delivered. The mechanical design for the primary and secondary mirror mounts has been completed, and they are now being fabricated. The optics testing procedure for the completed diagnostic is being developed. The presently anticipated date for fielding the diagnostic at NOVA is now in the early Fall of 1994.

OSA: Presently the diodes have been in operation for about two weeks. Typical SBS fluences detected near (but outside) the $f / 8$ cone of beam 7 from the Los Alamos large, gas-filled hohlraums are $\approx 100 \mathrm{~J} / \mathrm{sr}$. It is likely that some of this fluence is due to scatter driven by other beams. Tests in which the line of sight of some diodes was blocked during a few shots verified that the signals are coming from detection of light rather than from electrical-noise pickup. The time-resolved channels are coming into operation shortly, which should clarify the meaning of the measurements presently underway.

Axial Imager: With only a $527 \mathrm{~nm}$ rejection filter in place, the 
emission from a few Los Alamos large, gas-filled hoblraums (aleng the hohlraum axis) was imaged by the lens into a calorimeter. This was done to ascertain the filtration level appropriate for the gated detector. The detected scatter was typically only about $10 \mathrm{~J} / \mathrm{sr}$. The gated detector is being tested now, and SBS and $\omega_{p c}$ images should be available shortly.

\section{Acknowledgments}

It is a pleasure to acknowledge the excellent assistance in these three diagnostics provided by Los Alamos technicians, namely Scott Evans, John Jimerson and Tom Sedillo. Important guidance was received in many useful technical and managerial discussions held with Joe Mack, Robert Watt and Allan Hauer at Los Alamos, and with Greg Tietbohl, Sham. Dixit, Curt Laumann, Dee Pennington, Tim Weiland, Bob Wolfe, Howard Powell, Paul Wegner, Dave Eimerl, John Caird, Mark Henesian, John Miller, Don Campbell, Tom Crabtree, Dave Montgomery, Joe Kilkenny, Bob Kauffman, Héctor Baldis, Dick Berger, Brian McGowan and John Moody at Livermore. Pete Ludwigsen wrote the software that extracts the diode data. Bob Wolfe and Phil Armatis 
measured the spectral transmission of the turning mirror candidates for use in the FABS, and produced expected spectral response curves for various combinations. The role of Greg Tietbohl in coordinating or performing the extensive mechanical engineering at NOVA needed for these projects was crucial for their success. Greg also suggested the FABS name. The support of the Inertial Confinement Fusion office at Los Alamos to these efforts is gratefully acknowledged.

This work was funded by the US Department of Energy. 


\section{References}

[1] H. A. Baldis, E. M. Campbell, and W. L. Kruer. Laser-plasma interactions. In Handbook of Plasma Physics, Vol. 3: Physics of Laser Plasma, chapter 9, pages 361-434. North-Holland, Amsterdain, London, New York, Tokyo, 1991.

[2] S. M. Dixit, I. M. Thomas, B. W. Woods, A. J. Morgan, M. A. Henesian, P. J. Wegner, and H. T. Powell. Random phase plate for beam smoothing in the nova laser. Applied Optics, 32:2543-2554, 1993.

[3] Inc. Grant Applied Physics. Product descriptions. Grant Applied Physics Inc., 2011 Los Angeles Ave., Berkeley, CA 94707, Phone: (510)526-4683, FAX: (510)526-7611.

[4] Y. Kato, K. Mima, N. Miyanaga, S. Arinaga, Y. Kitagawa, N. Nakatsuka, and C. Yamanaka. Rpp. Physical Review Letters, 53:1057, 1984.

[5] J. H. Nuckolls, L. Wood, A. R. Thiessen, and G. B. Zimmerman. Indirect-drive icf. Nature, 239:139, 1972. 


\section{Figures}

Fig. 1. Illumination geometry of a typical NOVA hoblraum. Some optical diagnostics are indicated, with those improved, built, or being built by Los Alamos shown in a shaded box.

Fig. 2. The Full Aperture Backscatter Station (FABS).

Fig. 3. Top: OSA detector locations. Bottom: The fluence due to the probe beams and the heater beams can be resolved in time by the fiber-optic channels fed into a streak camera.

Fig. 4. Simplified illustration of the Axial Imager. 


\section{Fig. 1}

spatially-discriminating optical streaked calorimeter + spectrograph (SBS, SRS, $2 \omega_{\mathrm{po}}$ ) imager (SBS)

calorimeter + streaked optical spectrometer +

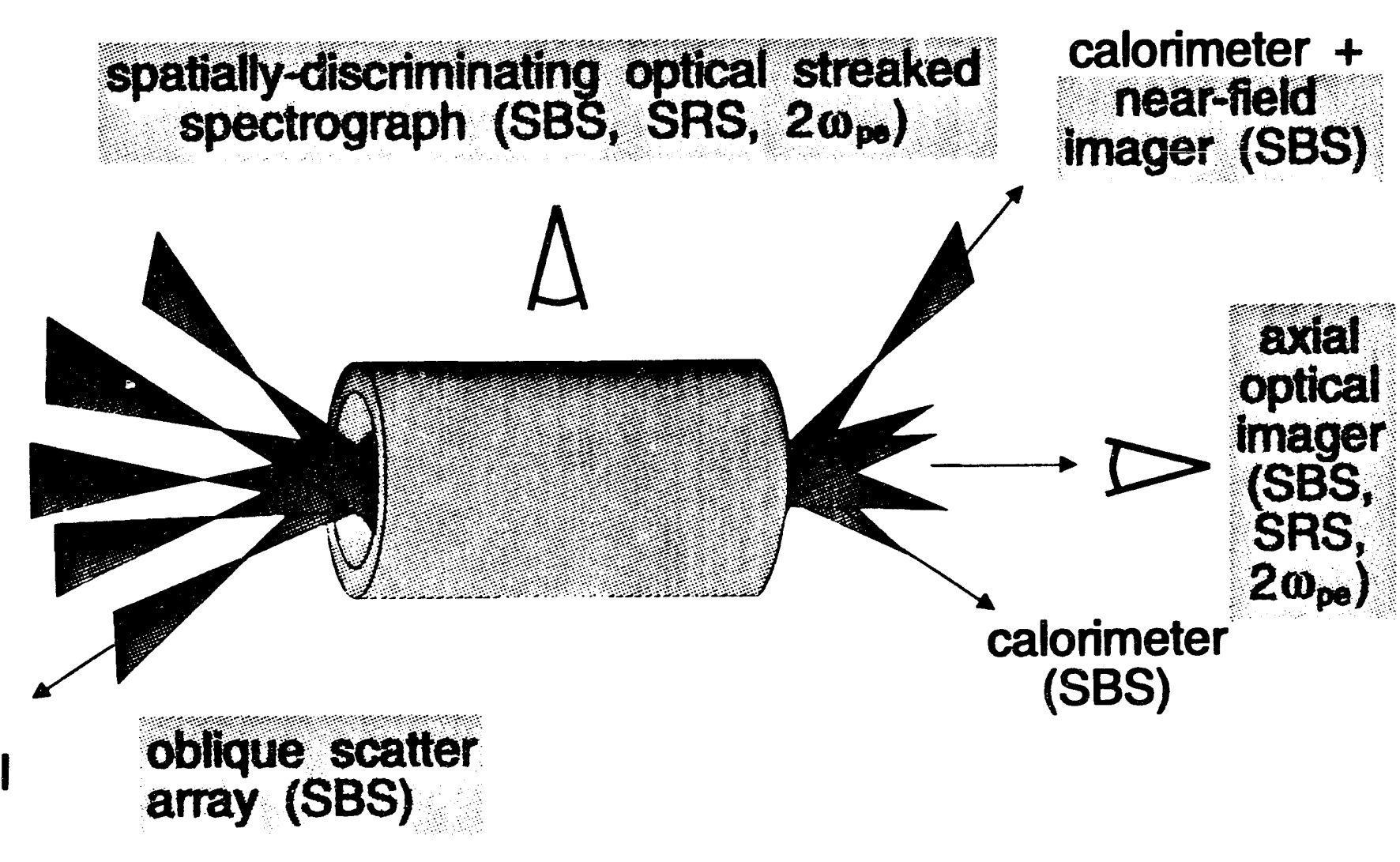

target Imager

(future; SBS, SRS) 


$$
\text { Fig. } 2
$$

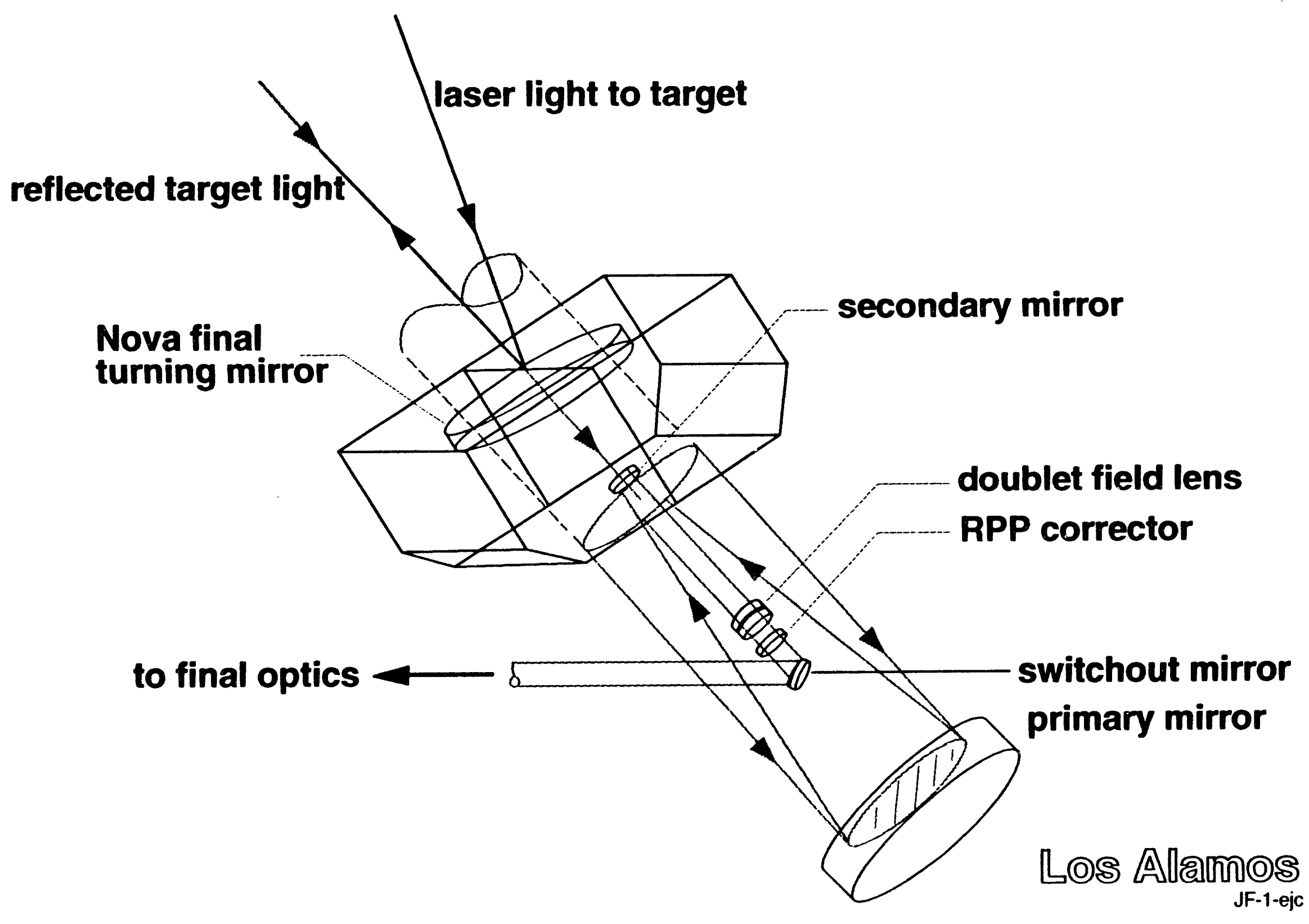




$$
\text { Fig. } 3
$$

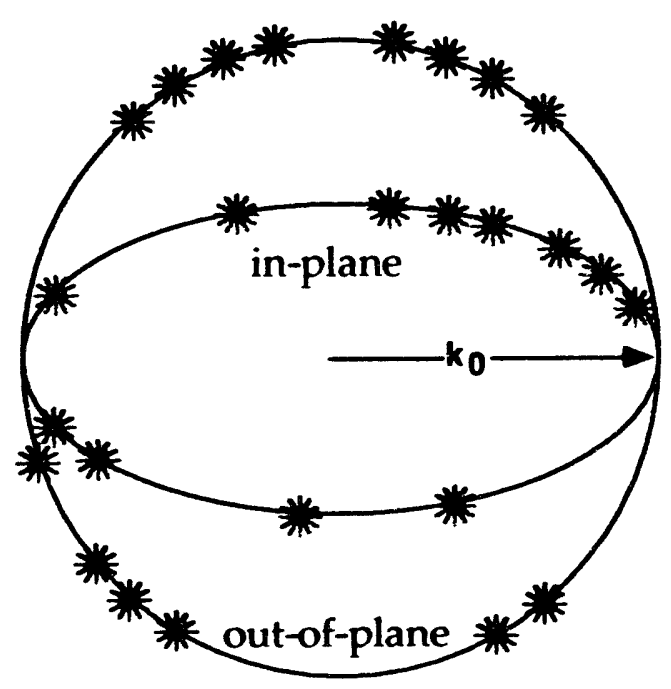

\&TOP

$$
\text { ByTOM } \rightarrow
$$

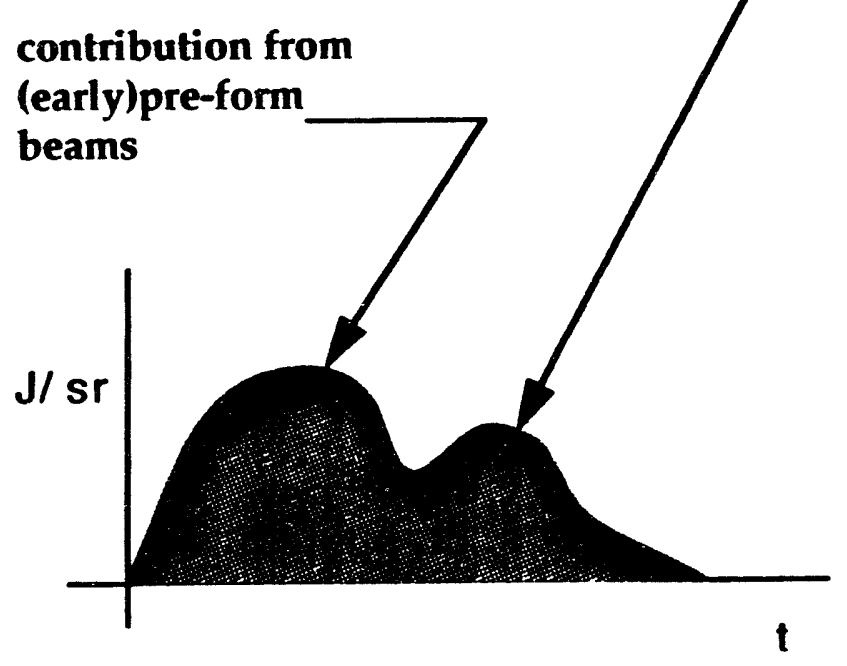

High Temp. '94/ K.S. Bradley; 1

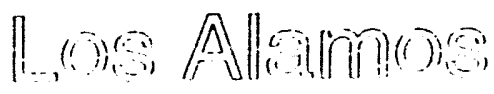




$$
\text { Fi6. } 4
$$

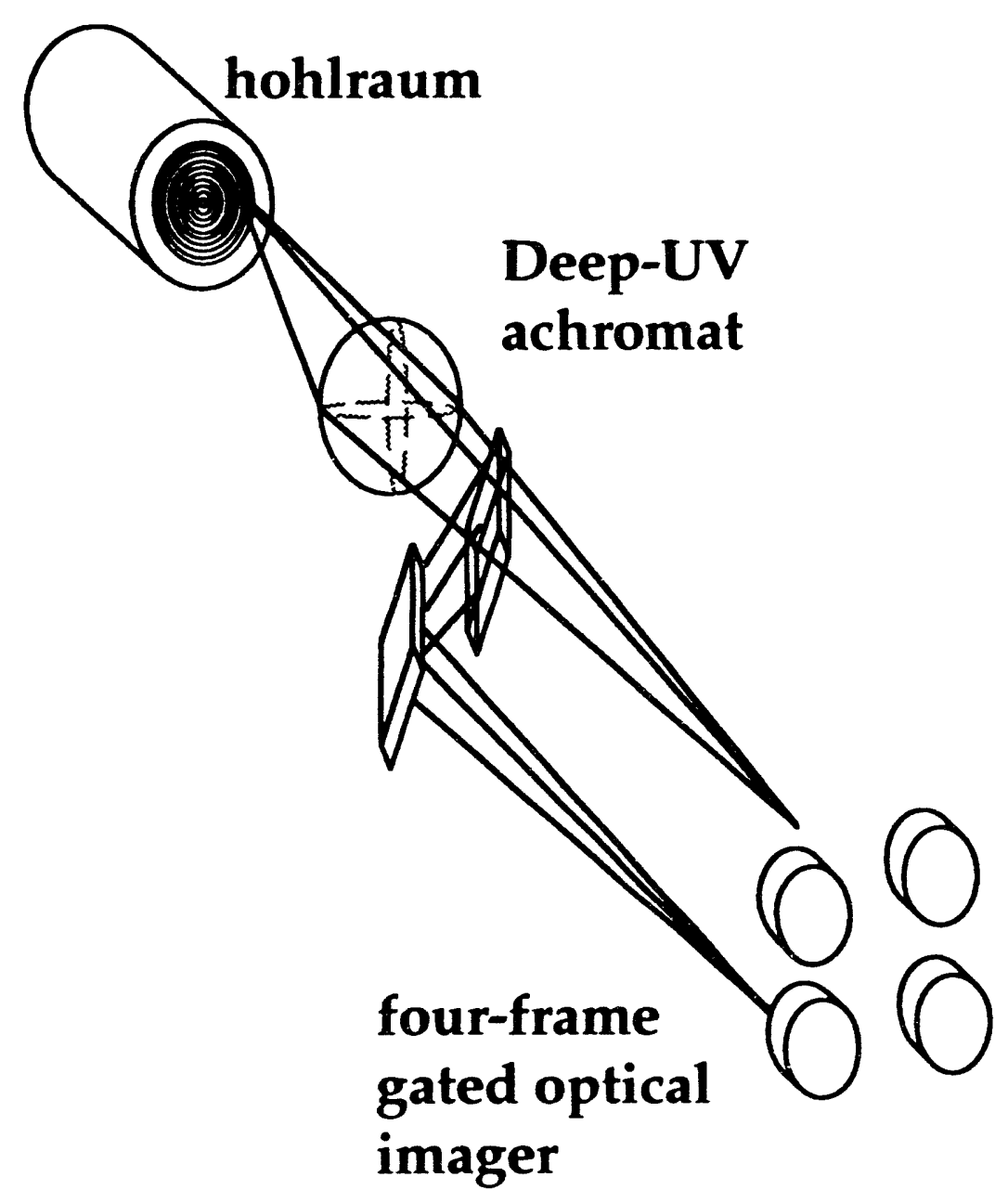

High Temp. ‘94/ K.S. Bradley; 2

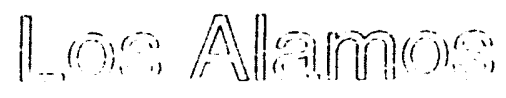



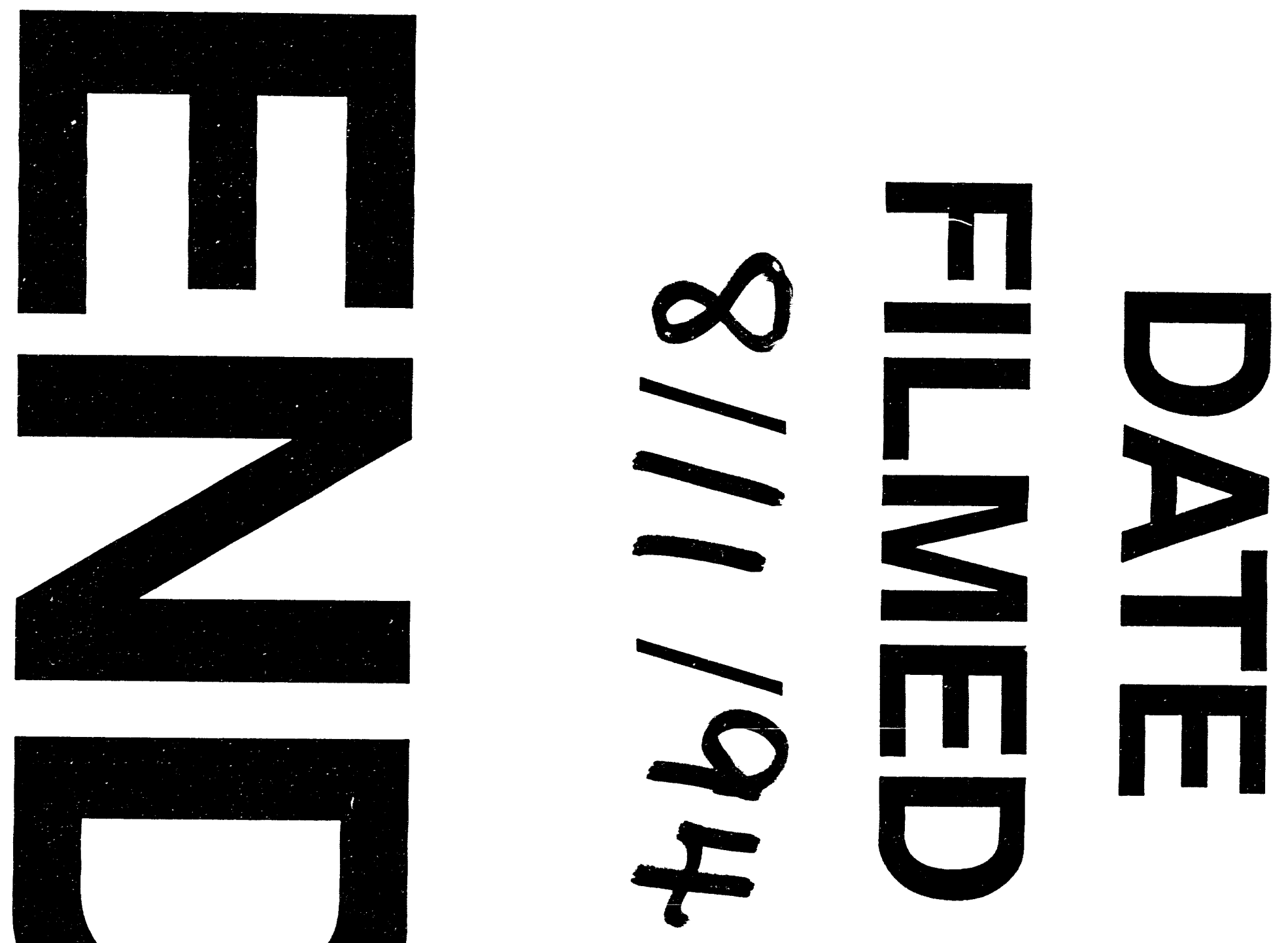

$\circlearrowright$
$\stackrel{0}{r}$
$H$ 
- 